\title{
EL CLUSTER TURÍSTICO DE UN DESTINO CULTURAL EN MÉXICO: SU CICLO DE VIDA Y LA RED DE ACTORES
}

\author{
Javier Gómez Diaz, \\ Julio Cesar Torres Valdez. \\ Andrés Enrique Miguel Velasco \\ Instituto Tecnológico de Oaxaca. México
}

\section{RESUMEN}

En este artículo, se analiza el grado de conformación del clúster del destino turístico de la ciudad de Oaxaca, México. Un elemento central en este ciclo es el grado de consolidación de las relaciones entre los actores que en él participan, por lo cual se analizan las redes sociales para determinar la fase del ciclo de vida del cluster. La hipótesis propuesta es que el grado de desarrollo del clúster de este destino es de gestación debido a la escasa vinculación entre los actores que lo conforman, la cual se confirma con los resultados empíricos que reflejan una red de baja densidad.

Palabras clave: cluster, ciclo de vida del cluster, redes sociales, Oaxaca, turismo.

\section{The cluster of a cultural tourism destination in México: life cycle and the network of actors}

\begin{abstract}
This article examines the degree of formation of the cluster of the tourist destination of the city of Oaxaca, Mexico. A central element in this cycle is the degree of consolidation of relations between the actors involved, by which we analyze the social networks to determine the stage of the life cycle of the cluster. The proposed hypothesis is that the degree of deve-

Recibido: 9 de mayo de 2015

Devuelto para su revisión: 5 de febrero de 2016

Aceptado: 14 de abril de 2016

División de Estudios de Posgrado e Investigación. Instituto Tecnológico de Oaxaca. Avenida Ing. Víctor Bravo Ahuja, nº 125. Esquina Calzada Tecnológico. C.P. 68030 OAXACA (México). E-mail: gomezdiazjavier@ hotmail.com, jcesartv@gmail.com, andres.miguel@itoaxaca.edu.mx
\end{abstract}


lopment of the cluster of this destination is of type pop-up due to a weak association between the actors that make up, which is confirmed with the empirical results that reflect a network of low density.

Keywords: cluster, cluster lifecycle, social networks, Oaxaca, tourism.

\section{INTRODUCCIÓN}

Si hubiese que buscar una característica que pueda diferenciar al turista del siglo XXI, ésta sería su creciente capacidad de elección, la cual está influida por el acceso a internet y tecnologías de la información. Reyes y Barrado (2005:37) explican que el turista de la era del conocimiento y la información tiene a su alcance una enorme gama de ofertas turísticas y de ocio; así como, una casi ilimitada capacidad para hacerlas efectivas por el aumento del tiempo libre, la continua mejora de los medios de transporte y la introducción de internet en las formas de acceso a la comunicación, distribución y comercialización turística. Lo que implica un gusto creciente por una oferta más especializada y discriminada en nuevas líneas de producto (Exceltur, 2007).

Estos nuevos productos son conformados por la conjugación de las ventajas comparativas (atractivos naturales, culturales, gastronómicos, etc.) y las ventajas competitivas: la gestión estratégica de la información, la capacidad de innovación, conocimiento, etc. Uno de los paradigmas más empleado para desarrollar estos nuevos productos es la "estrategia clúster", la cual ha tomado importancia, debido a que contribuye a conformar productos creados por los prestadores de servicios más competitivos y de mayor calidad, con identidad propia lo que llama la atención de los turistas (Iordache, Ciochina y Asandei, 2010:2010 y Dragusin, Lumina y Petrescu 2010:289).

El cluster, como un mecanismo de aglomeración permite generar ventajas competitivas a través de la generación de conocimientos, contribuye al impulso de innovaciones, con lo cual se incrementa la competitividad. Es una herramienta que se está impulsando como estrategia, que permite a las empresas, actividades, regiones y ciudades, competir en diferentes escenarios económicos.

La vinculación de los actores de la actividad turística a través de las redes sociales que se van conformando alrededor de la actividad, permite visualizar su consolidación en las regiones o localidades donde ésta se desarrolla, lo cual puede incidir en la consolidación del cluster. Tomando como referencia esta temática, el objetivo del presente artículo es analizar el grado de conformación del clúster del destino turístico de la ciudad de Oaxaca, México. La hipótesis que se propone plantea que el grado de desarrollo del clúster de este destino turístico es de gestación, debido a la escasa vinculación entre los actores que lo conforman.

\section{MARCO CONTEXTUAL: EL DESTINO TURÍSTICO DE LA CIUDAD DE OAXACA}

El trabajo de investigación se desarrolló en el destino turístico de la ciudad de Oaxaca, México (figura 1). Este destino ostenta la categoría de ciudad patrimonio cultural de la 
humanidad, ya que posee tres sitios en la lista de patrimonio mundial como son: centro histórico de Oaxaca, zona arqueológica de Monte Albán y las cuevas prehistóricas de Yagul y Mitla. Este destino tiene un amplio patrimonio edificado, centros culturales (26 monumentos religiosos donde sobresalen Santo Domingo Guzmán, La Catedral, La Soledad, etc; 10 bibliotecas; 11 museos, etc.), una rica producción artesanal (destacando la alfarería en barro negro, rojo y verde, alebrijes, entre otros muchos) y múltiples fiestas tradicionales de las cuales la Guelaguetza es un icono representativo de sus tradiciones. Lo que le brinda ventajas comparativas para desarrollar actividades turísticas con un enorme potencial. Cabe señalar que la actividad económica del turismo es la principal fuente de ingresos para las familias que habitan el destino.

\section{Figura 1 \\ DESTINO TURÍSTICO CIUDAD DE OAXACA}

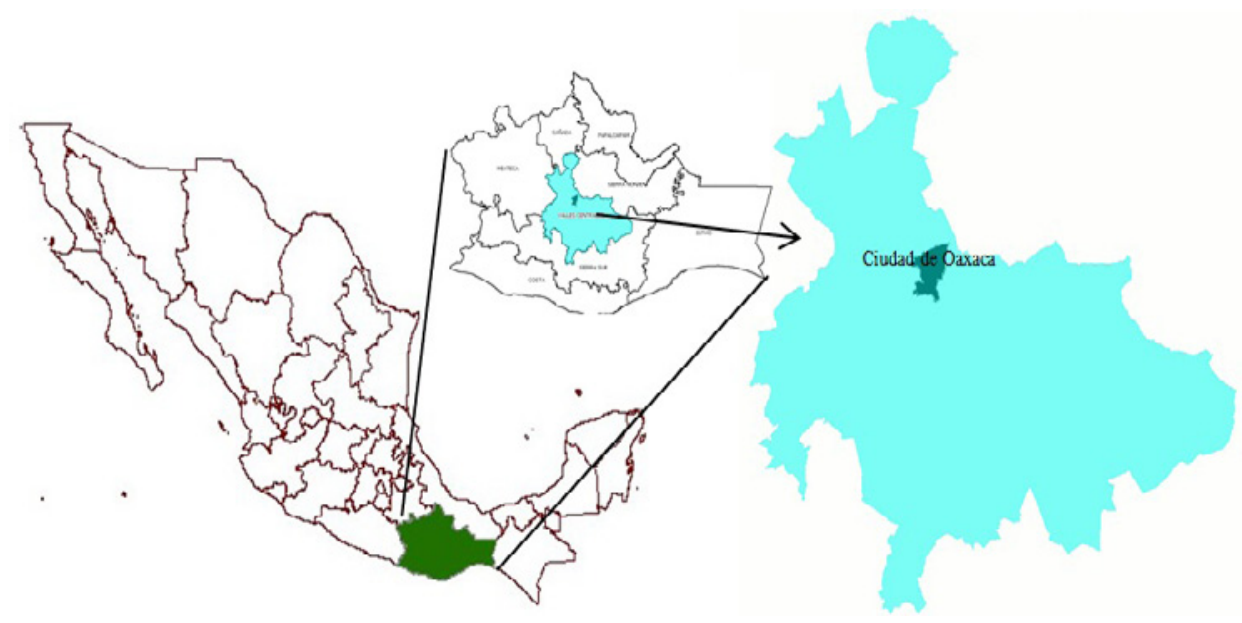

Fuente: elaboración propia.

Desde el punto de vista turístico, la ciudad de Oaxaca, es un destino colonial muy importante en México, ofrece a sus visitantes cinco rutas turísticas regionales denominadas: 1) Dominica 2) Sierra Juárez, 3) Mitla, 4) Ocotlán y 5) Monte Albán-Zaachila. En estas los visitantes pueden apreciar: atractivos culturales (iglesias con arquitectura virreinal, zonas arqueológicas, etc.) atractivos naturales (Hierve el agua, árbol del tule, bosques de exuberante vegetación, etc.) (Oaxaca-mío, 2000). Las fiestas más importantes en este destino son: Semana Santa, Guelaguetza y Noche de Rábanos.

Madrid y Cerón (2013) mencionan que la etapa del ciclo de vida del destino turístico propuesto por Butler (1980), en la que se encuentra el destino turístico de la ciudad de Oaxaca es de consolidación, con una notable infraestructura, así como una imagen y buen posicionamiento de los atributos del destino en el conjunto de destinos coloniales (Filgueiras, 2009). La oferta turística está conformada por pequeñas y medianas empresas (PyMES), principalmente familiares. La dinámica de las empresas muestra que durante 2004 y 2012 
existió un crecimiento en el sector hotelero y restaurantero (18 y $40 \%$ respectivamente), contrario a las agencias de viaje y guías de turistas los cuales decrecieron en un $4.5 \%$ y $26 \%$ respectivamente (Sistema de información turística estatal/SECTUR Oaxaca, 2012).

Como se observa en la figura 2 la afluencia de turistas a este destino, es mayoritariamente proveniente del mercado nacional y está conformada por los viajeros provenientes de los estado de Puebla, Veracruz y Distrito Federal, los cuales integran el $50.27 \%$. El mercado internacional está representado por los turistas provenientemente de América del Norte y Europa con el 21 y $29 \%$ respectivamente, sin embargo en los últimos años Australia se ha posicionado como un mercado potencial generando un flujo constante hacia este destino (Sistema de información turística estatal/SECTUR Oaxaca, 2012).

\section{Figura 2 \\ AFLUENCIA DE VISITANTES EN EL DESTINO \\ TURÍSTICO DE LA CIUDAD DE OAXACA}

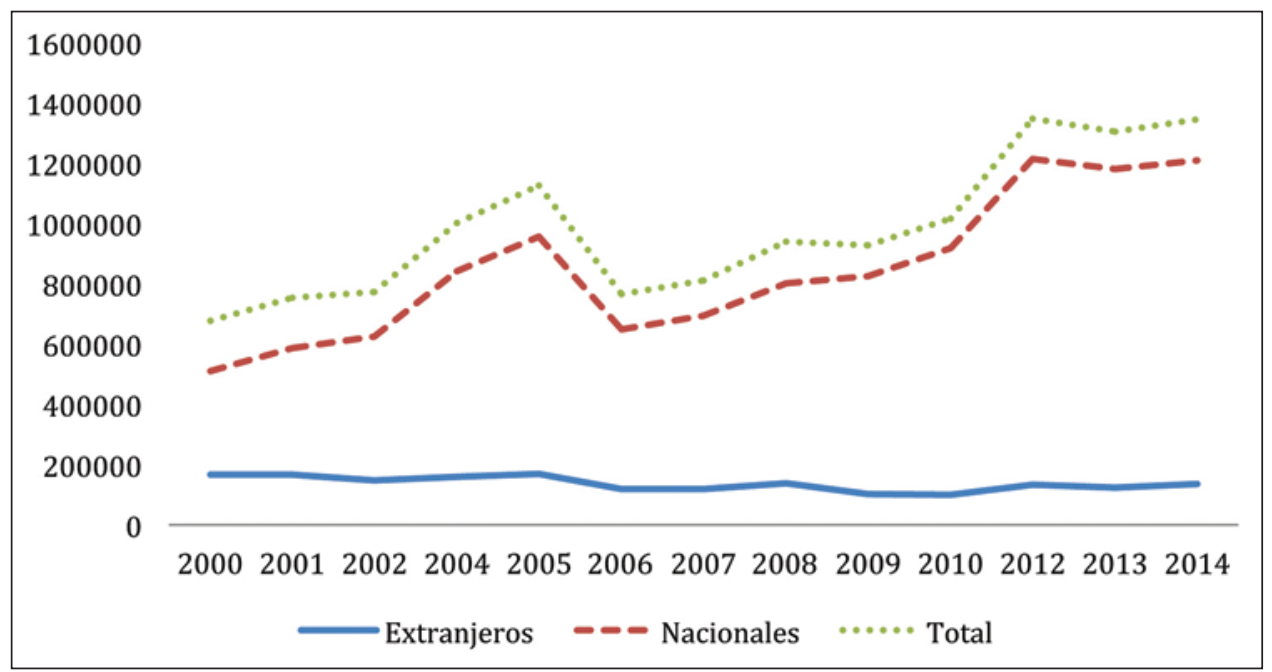

Fuente: elaboración propia.

En el conjunto de destinos coloniales de México en el periodo 1986-2008, en materia de afluencia turística este destino ha figurado entre los primeros tres lugares (Puebla, Mérida y Oaxaca), sin embargo, a partir del 2009 Oaxaca pasó a ocupar el cuarto lugar siendo desplazado por la ciudad de Querétaro. Su tasa de participación en el segmento cultural es de apenas el $1.37 \%$ en año 2014.

Este comportamiento es resultado de que a partir del 2006, el entorno social de este destino se caracteriza por una alta incidencia de movimientos políticos sociales. Así tenemos que en los años 2012 y 2013, se realizaron 982 y 2375 marchas y plantones respectivamente (COTAIPO, 2014), lo que genera afectaciones al turista que visita al destino, así como incertidumbre de las actividades a desarrollar por parte de los prestadores de servicios turísticos. 


\section{MARCO TEÓRICO: EL CLÚSTER TURÍSTICO, SU CICLO DE VIDA Y LA RED DE ACTORES}

El clúster se define como un conjunto de actividades poco diferenciadas, concentradas en un área geográfica, ofreciendo servicios de calidad, con cohesión política y social mediante la coordinación efectiva de la cadena de producción y la adopción de una excelente gestión de la red de empresas, que generan ventajas comparativas y competitivas (Iordache et al., 2010:105). Particularmente, el clúster de turismo es entendido "como una forma de organizar la actividad turística de una región articulando los diversos actores locales (población, empresarios, gobierno, ONGs) logrando que interactúen, cooperen, aprendan y generen conocimiento. Surgen como alternativa para proporcionar el desarrollo socioeconómico a nivel local" (Mancildo, Rejame y Segre, 2010:820).

El estudio del fenómeno cluster muestra que los cluster tienen tres dimensiones básicas: la proximidad geográfica, las redes entre las empresas y las redes con organismos e instituciones gubernamentales y educativas (Toledo, Quelopana y Castroman 2006; Cuervo, Montoro y Romero, 2009; Iordache et al. 2010).

La dimensión geográfica se refiere a la proximidad de la ubicación de las empresas y es la dimensión del clúster que sólo se incluye en la mayoría de los estudios cuantitativos. El aspecto geográfico es clave en la definición de un cluster (Acuña, 2000; Porter, 2003; Iordache et al., 2010; Ivanovic et al., 2010).

Los cluster indican un caso especial de empresas en red que están concentradas geográficamente (Ferreira y Estevao, 2009). Las cuales pueden tener relaciones formales o informales. Las formales se relacionan con la dependencia con el mercado. Estas consisten en fuertes vínculos productivos y comerciales. Las relaciones de interdependencias no negociables toman la forma de convenciones, reglas informales, y los hábitos que coordinan los actores económicos en condiciones de incertidumbre.

Por último, la tercera dimensión, las redes institucionales, se refieren a las relaciones entre empresas, organizaciones no gubernamentales y las organizaciones gubernamentales dentro del cluster (Porter, 1998; Acuña, 2000; Cuervo et al., 2009; Iordache et al., 2010).

El ciclo de vida del cluster se produce como interacción entre las dimensiones industrial, regional y relacional del mismo (Crespo, 2011:2030). Menzel y Fornahl (2010) explican que, el ciclo de la actividad afectará al ciclo de vida del cluster, pero no lo determina. Por ejemplo en una actividad en declive puede haber clusters que declinen mientras otros se renuevan y transforman para empezar un nuevo ciclo de crecimiento. Ello explica que dos clusters en la misma actividad puedan seguir trayectorias diferentes en el mismo momento del tiempo.

Esta forma de evolucionar del cluster de diferente manera en la misma actividad está relacionada con el grado de desarrollo de los elementos que participan en las etapas del ciclo de vida. En el cuadro 1 se mencionan los principales elementos del ciclo de vida de acuerdo a diferentes autores, es de notarse que un elemento primordial es el nivel de consolidación de las relaciones entre los actores que conforman el cluster. 


\title{
Cuadro 1 \\ ELEMENTOS QUE CALIFICAN LAS ETAPAS DEL CICLO DE VIDA DEL CLUSTER
}

\begin{abstract}
Etapa del ciclo
de vida del

cluster

Elementos que califican cada etapa

Gestación

Existen ventajas comparativas pero las industrias y empresas existentes no están vinculadas, las empresas aprovechan la oportunidad de negocio

Emergencia

Las industrias, empresas e instituciones tienen vínculos incipientes, generando

y aprovechando oportunidades que le brindan las ventajas comparativas. No existe una masa critica lo que limita las fuerzas de aglomeración, el sector económico se encuentra en la fase de emprendimiento

$\begin{array}{ll}\text { Expansión } & \text { complementarse, se desarrolla una imagen, con una connotación de la actividad } \\ \text { de negocio que se desarrolla, el sector económico se encuentra en la fase del }\end{array}$

Las industrias, empresas e instituciones presentan vínculos sólidos para desarrollo de infraestructura competitiva

Existen redes colaborativas de industrias, empresas e instituciones vinculadas

Maduración a su ecosistema en donde se fomenta la circulación de información y conocimiento a través de colaboraciones informales lo que conlleva a la constante innovación
\end{abstract}

Fuente: elaboración propia con información de Rosenfeld, 1997; Örjan, 2008; Guerra y Daniel, 2010 y Crespo, 2014.

Guerra y Daniel, (2010) mencionan que las etapas del ciclo de vida del cluster se pueden agrupar en tres procesos que se dan durante el ciclo de vida del cluster: integración, desarrollo y consolidación (figura 3). Estos procesos suceden de dos maneras diferentes; ya sea por la ocurrencia de eventos debidos a una evolución natural de los componentes del cluster, o bien, como resultado de la implementación de acciones deliberadas orientadas a la formación balanceada de estos componentes, de tal forma que partiendo de una masa crítica de empresas, industrias e instituciones se llegue a tener un cluster consolidado (Guerra y Daniel, 2010:19).

Un indicador de la etapa del ciclo de vida del clúster, son los vínculos o redes que el mismo logra fomentar y consolidar. Una red se define como un conjunto estrecho de vínculos explícitos, selectos y con patrones preferenciales que se dan en un conjunto de empresas e instituciones a partir de ventajas complementarias y relaciones de mercado, teniendo como principal meta la reducción estática y dinámica de la incertidumbre (García y Lara, 2004:150).

Un hilo común, tanto en la literatura económica y sociológica es la noción de los vínculos locales que forman la base geográfica que definen a un clúster. Estos vínculos tienden más a menudo a basarse en las transacciones comerciales en los análisis económicos. En la sociología económica se hace más hincapié sobre la importancia de los vínculos no comerciales los cuales están basados en el tema de la amistad, lazos familiares, etc. En ambos casos, por definición, "se dice que existe un cluster en virtud de la existencia 
de vínculos a nivel local confinados" (Eisingerich, Bell, Tracery, 2010). Estos vínculos pueden ser de negocios y de intereses públicos, centrados en el pequeño espacio geográfico donde los contactos físicos están disponibles y la dinámica de estos vínculos cambia continuamente (Dragičević, 2006).

\section{Figura 3 \\ PROCESOS QUE OCURREN EN EL CICLO DE VIDA DEL CLUSTER}

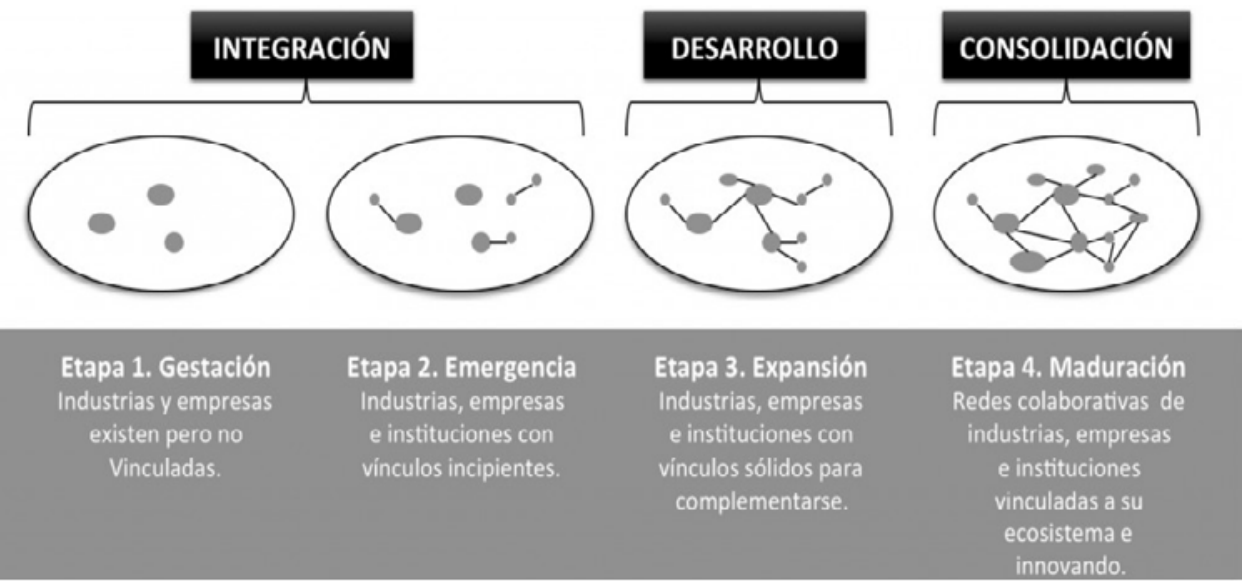

Fuente: Guerra y Daniel, 2010.

Eisingerich et al., (2010:241) menciona que las redes sociales son especialmente importantes para los clusters en donde los vínculos en la red, si son fuertes, facilitan la transferencia y asimilación de los conocimientos dentro de los grupos, y la apertura hacia nuevas redes, la cuales se supone proporcionan a los miembros del clúster el acceso a nuevos conocimientos y formas de operar. La fuerza de la red y la apertura de la misma presentan una correlación positiva con el rendimiento del clúster. De hecho, estas características de la red podría decirse que constituyen los bloques de construcción de la ventaja competitiva en los clusters. Sin embargo, también las relaciones entre la apertura de la red, la fuerza de la red y el rendimiento del clúster puede variar de acuerdo a la incertidumbre del medio ambiente, a medida que los entornos sean más inciertos, la importancia relativa de la apertura de la red aumenta el rendimiento del clúster, mientras que la importancia relativa de fuerza de la red disminuye.

Las redes son importantes porque: a) reducen la incertidumbre tecnológica y de mercado b) generan ganancias cuando la cooperación técnica es exitosa, c) reducen comportamiento oportunista al promover entre los participantes el desarrollo de lenguaje común, entendimiento, compromisos mutuos y confianza, d) coadyuvan a disminuir complejidad y naturaleza intersectorial de nuevas tecnologías; e) permiten la expansión de la producción y entrada de nuevos procesos de trabajo, f) se obtiene información y conocimientos que contribuyen a monitorear el ambiente, reducir el ciclo de vida de productos o facilitar la entrada a nuevos mercados y g) crean y establecen un conjunto de estándares, normas, 
reglas, sistemas y especificaciones tecnológicas que disminuyen costos de administración y de producción (Garcia y Lara 2004, Bobby, 2009, Cuervo et al., 2009 y Cai y Fan, 2011).

En el análisis de redes se describen y estudian las estructuras relacionales que surgen cuando diferentes organizaciones o individuos interaccionan, se comunican, coinciden, colaboran, etc., a través de diversos procesos o acuerdos, que pueden ser bilaterales o multilaterales; de este modo la estructura que emerge de la interrelación se traduce en la existencia de una red social (Sanz, 2003 p 23.).

La idea básica del enfoque de redes es que los individuos son actores intencionales con motivaciones sociales y económicas, cuyas acciones están influenciadas por una red de relaciones en las cuales están insertos. Así, los actores y las relaciones que mantienen entre ellos forman una red social, siendo un elemento clave la posición que cada actor ocupa en ella, lo que forma la estructura general de la red, la cual, a su vez, implica oportunidades y restricciones para los actores (Casas, Dettmer, Celis y Hernández, 2007).

De acuerdo con Márquez, González, Agra, Vega, Pinto y Quiroga (2013), el Análisis de Redes Sociales (ARS) tiene la capacidad para entender las redes desde distintas perspectivas considerando como más relevantes, el estudio de las propiedades de la estructura y de la localización de la red, así como los roles adquiridos atendiendo a la posición en la red.

De acuerdo a Santos (2010), los dos aspectos fundamentales de las redes sociales son su estructura y su contenido. La estructura se refiere al patrón que emerge de los lazos sociales entre actores. Dos propiedades importantes en la estructura de las redes sociales son la centralidad de un actor (cuán estratégicamente ubicado está un actor en la red) y la cohesión estructural (cuán vulnerable es una red social a fragmentarse). En la centralidad, un actor central puede tener un mejor acceso a la información que actores periféricos dada su posición estratégica en la red social. En relación con la cohesión estructural, las redes sociales con alta cohesión estructural propician mejores condiciones para la difusión de información debido a que ningún actor particular puede controlar su flujo.

Por su parte, el contenido se refiere al tipo de relación social que vincula a los actores en una red social (por ejemplo, amistad, parentesco, relación laboral). El tipo de relación social (condiciona los recursos que pueden circular en la red.

\section{METODOLOGÍA}

\subsection{Fuentes de información}

La metodología del presente artículo retoma la propuesta de Merino y Pulido (2009), la cual parte del reconocimiento de la importancia de las relaciones entre los actores turísticos de un determinado territorio. Este planteamiento se centra en identificar los actores que intervienen durante el proceso y evalúa las relaciones que se establecen entre ellos. Esta metodología está compuesta de diferentes pasos, sin embargo, se consideraron únicamente los pasos necesarios para cumplir con el fin de este trabajo los cuales fueron: 1) selección de informantes clave, 2) identificación previa de los actores turísticos y análisis de sus principales características, 3) identificación de actores con los que se interrelacionan, 4) análisis de las características básicas y 5) componentes de las relaciones y análisis de la estructura formal de la red de actores. 
Siguiendo esta estructura metodológica se realizaron tres fases para cumplir el objetivo planteado:

a) Primero se participó en foros con los cuales se buscó identificar e interactuar con los actores que conforman el cluster turístico, estos foros son: el Foro para el Desarrollo del Turismo Sustentable de Oaxaca (2014) y el Panel de Opinión de Empresarios Oaxaqueños Relacionados con el Arte, la Cultura y el Turismo (2014), Consejo Honorario de Participación Ciudadana del Municipio de Oaxaca (2014), Expo-PyME (2013 y 2014), Expectativas, Satisfacción y Fidelidad del Turista en los Hoteles y Restaurantes de la Ciudad de Oaxaca (2014), Desarrollo del Cluster Turístico de Oaxaca (2014)

b) Posteriormente, se realizaron entrevistas al sector del gobierno relacionado con el turismo (Secretaria de Turismo y Desarrollo Económico (STyDE) y Secretaría de Turismo del Municipio de Oaxaca de Juárez), también se entrevistó a organizaciones propias del sector (Asociación Mexicana de Hoteles y Moteles A.C., la Unión de Guías de Turistas de Oaxaca, Cámara Nacional de la Industria de Restaurantes y Alimentos Condimentados, etc. (CANIRAC) ) así, como a instituciones educativas del área turística (Universidad Autónoma Benito Juárez de Oaxaca (UABJO), Universidad del Mar (UMAR), Instituto Tecnológico de Oaxaca (ITO), Instituto Universitario de Oaxaca (IUO), Universidad del Golfo, etc.)

c) Como último paso, se realizaron entrevistas y se aplicaron encuestas al sector empresarial (hoteles, restaurantes, agencias de viaje y guías de turistas) para conocer las relaciones que se establecen con el resto de los actores.

La información fue analizada en el programa Ucinet 6 versión 6.232 (Borgatti, Everett y Freeman, 2002) para Windows, con en el cual se realizó el análisis de redes sociales del cluster turístico. Este programa permite visualizar de manera gráfica las características de la red de actores como son la densidad, el grado de centralidad, el índice de centralización y grado de cercanía de los vínculos de las propias redes. La densidad de la red es una propiedad que se refiere al volumen de interconexiones y esta constituida por la proporción de conexiones efectivamente existentes sobre la totalidad de conexiones posibles. La centralidad muestra en forma genérica la existencia de miembros que ocupan posiciones que varían en el grado de centralidad o periferia usando el volumen de conexiones para cada miembro de la red, (Vivas, 2001). El índice de centralización, es una condición especial en la que un actor ejerce un papel claramente central al estar conectado con todos los nodos, los cuales necesitan pasar por el nodo central para conectarse con otros. Grado de cercanía, es la capacidad de un nodo de llegar a todos los actores de una red (Velásquez y Aguilar, 2005).

\subsection{Procedimiento de análisis de las redes sociales}

En el presente artículo se evalúan la densidad de la red, el índice de centralización, el grado de centralidad y grado de cercanía de las redes para evaluar el ciclo de vida del cluster. En el primer aspecto, se toma como base la "densidad de la red", que es una medida 
de cohesión de una red social y se refiere fundamentalmente a la cantidad de relaciones observadas en relación a la cantidad de relaciones posibles (Williner, Sandoval, Frias y Pérez, 2012). Este parámetro determina el grado de similaridad de las opiniones, apreciaciones y creencias comunes a un grupo social. Este valor se presenta expresado en una tasa.

$$
D=\frac{r}{n * 100}
$$

Donde D: densidad; r: número de vínculos establecidos entre los actores; n: número vínculos totales que pudieran existir. La magnitud de esta densidad puede evaluarse por la escala indicada en la Tabla 2.

Tabla 2

DENSIDAD DE LA RED Y SU GRADO DE CONECTIVIDAD

\begin{tabular}{|l|l|}
\hline Densidad (porcentaje) & Grado de conectividad \\
\hline $0-20$ & Muy baja \\
\hline $21-40$ & Baja \\
\hline $41-60$ & Medio \\
\hline $61-80$ & Alto \\
\hline $81-100$ & Muy alto \\
\hline
\end{tabular}

Fuente: elaboración propia.

El "índice de centralización” es una condición especial en la que un actor ejerce un papel claramente central al estar conectado con todos los nodos, los cuales necesitan pasar por el nodo central para conectarse con otros (figura 4). El "índice de centralización" de una red, ya sea el de entrada o el de salida, indica qué tan cerca está la red de comportarse como una red estrella, en donde un actor juega un papel central que controla a toda la red; o qué tan lejos está de ese comportamiento, el cual es más favorable debido a que esto nos habla de una red bien conectada (Williner et al., 2012).

El "grado de centralidad" se divide en grado de entrada y grado de salida, los cuales dependen de la dirección del flujo. El grado de salida es la suma de las relaciones que los actores dicen tener con el resto. El grado de entrada es la suma de las relaciones referidas hacia un actor por otros. En el gráfico 5 vemos una red con forma de estrella (diagrama a), en la cual no cabe duda que el nodo 1 tiene la mayor centralidad posible de esta red. Es decir, posee el máximo grado y está lo más cerca posible de la mayor cantidad de nodos de la red. Contrario en lo que sucede en el diagrama b, en donde el nodo 1 no tiene la mayor centralidad de la red dada su posición. En el diagrama c, todos los nodos tienen el mismo grado de centralidad por el tipo de red que conforman. Es de notarse que en estas redes pequeñas es muy fácil identificar la posición de los nodos, sin embargo en redes más complejas es necesario el cálculo de centralidad con alguna herramienta de análisis de redes sociales. 


\section{Figura 4 \\ GRADO DE CENTRALIZACIÓN}

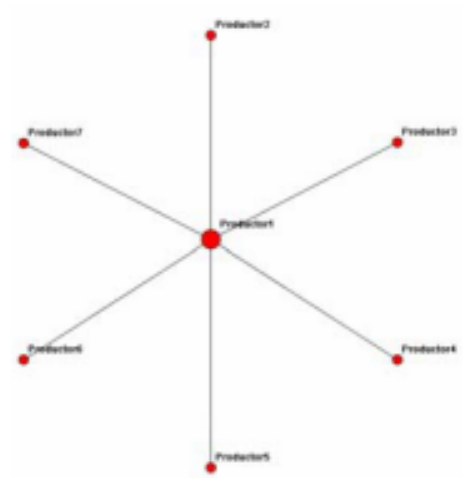

A) Red Estrella. Grado de centralización $=100 \%$

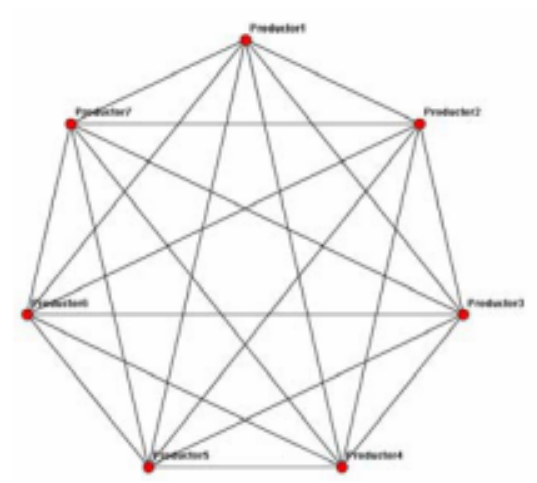

B) Grado de centralización $=0 \%$

Fuente: Velásquez y Aguilar, 2005.

Figura 5

EJEMPLO DE GRADOS DE CENTRALIDAD

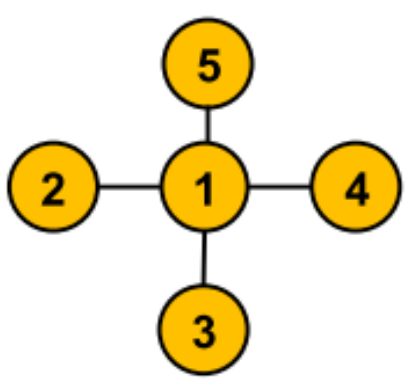

a)

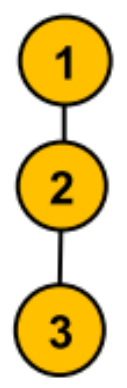

b)

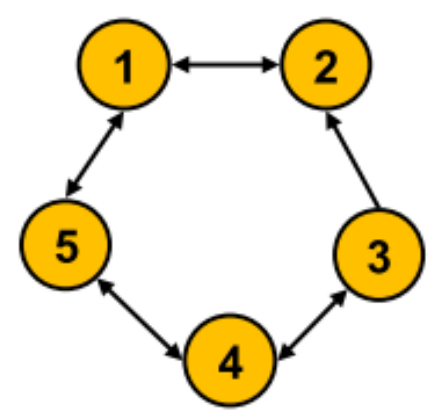

c)

Fuente: Williner et al., 2012.

Por otra parte, el "grado de cercanía" es la capacidad de un nodo de llegar a todos los actores de una red, este se calcula al contar todas las distancias geodésicas de un actor para llegar a los demás (Velásquez y Aguilar, 2005). Cabe mencionar que valores altos de cercanía indican una mejor capacidad de los nodos para conectarse con los demás actores de la red.

Ambos indicadores permiten visualizar el ciclo de vida del cluster. Una escala de esta medición se indica en la Tabla 3. 
Tabla 3

REDES SOCIALES Y CICLO DE VIDA DEL CLUSTER

\begin{tabular}{|l|l|l|}
\hline $\begin{array}{c}\text { Densidad } \\
\text { (porcentaje) }\end{array}$ & Grado de conectividad & Etapa del ciclo de vida del cluster \\
\hline $0-20$ & Muy baja & Gestación \\
\hline $21-40$ & Baja & Emergencia \\
\hline $41-60$ & Medio & Expansión \\
\hline $61-80$ & Alto & Maduración \\
\hline $81-100$ & Muy alto & Consolidación/Transformación* \\
\hline
\end{tabular}

*Consolidación/Transformación: es la etapa de plena madurez, y el fomento a la creación de nuevos cluster, enfocándose a otras actividades o simplemente adaptarse a las nuevas formas del mercado.

Fuente: elaboración propia.

\section{RESULTADOS}

En el destino de la ciudad de Oaxaca existen diversos actores turísticos que cooperan para impulsar el destino, en el cuadro 4 se pueden observar los principales actores y con quienes interactúan. Con quien se relacionan depende de los objetivos que busquen cada uno de los actores, así como la percepción que se tenga los otros actores basados en el tema de la confianza.

\section{Cuadro 4 \\ RELACIONES ENTRE LOS ACTORES DEL DESTINO TURÍSTICO DE LA CIUDAD DE OAXACA}

\begin{tabular}{|c|c|c|c|c|c|}
\hline \multirow[b]{2}{*}{ Actor } & \multicolumn{5}{|c|}{ Con quien se relaciona } \\
\hline & Gobierno & $\begin{array}{c}\text { Instituciones } \\
\text { educativas }\end{array}$ & Asociaciones & $\begin{array}{c}\text { Empresarios } \\
\text { de su mismo } \\
\text { segmento }\end{array}$ & $\begin{array}{c}\text { Empresarios } \\
\text { de otro } \\
\text { segmento }\end{array}$ \\
\hline CANIRAC* & $\mathrm{X}$ & $\mathrm{X}$ & $\mathrm{X}$ & $\mathrm{X}$ & \\
\hline $\mathrm{AMHMO}^{* *}$ & $X$ & $\mathrm{X}$ & & $\mathrm{X}$ & \\
\hline $\begin{array}{l}\text { Asociación Turística } \\
\text { de Oaxaca }\end{array}$ & & & & $\mathrm{X}$ & \\
\hline $\begin{array}{l}\text { Asociación de Guías } \\
\text { de Turistas }\end{array}$ & $\mathrm{X}$ & $X$ & $\mathrm{X}$ & & \\
\hline STyDE & & $\mathrm{X}$ & $\mathrm{X}$ & & \\
\hline $\begin{array}{l}\text { Secretaria de Turismo } \\
\text { Municipal }\end{array}$ & & $\mathrm{X}$ & $\mathrm{X}$ & & \\
\hline CECAT-UMAR $^{* * *}$ & $\mathrm{X}$ & & & & \\
\hline ICAPET $^{\dagger}$ & $\mathrm{X}$ & & & & \\
\hline CEC-IPN & $\mathrm{X}$ & & & & \\
\hline
\end{tabular}




\begin{tabular}{|l|l|l|l|l|l|}
\hline Hoteleros & $\mathrm{X}$ & $\mathrm{X}$ & $\mathrm{X}$ & $\mathrm{X}$ & $\mathrm{X}$ \\
\hline Restaurante & $\mathrm{X}$ & $\mathrm{X}$ & $\mathrm{X}$ & $\mathrm{X}$ & $\mathrm{X}$ \\
\hline Agencias de viaje & $\mathrm{X}$ & $\mathrm{X}$ & $\mathrm{X}$ & $\mathrm{X}$ & $\mathrm{X}$ \\
\hline Guías de turistas & $\mathrm{X}$ & $\mathrm{X}$ & $\mathrm{X}$ & $\mathrm{X}$ & $\mathrm{X}$ \\
\hline
\end{tabular}

* Cámara nacional de la industria de restaurantes y alimentos condimentados, ** Asociación Mexicana de Hoteles y Moteles de Oaxaca, $\square$ Secretaria de Turismo y Desarrollo Económico *** Centro de Capacitación Turística de la Universidad del Mar, $†$ Instituto de Capacitación y Productividad para el Trabajo del Estado de Oaxaca, $\$$ Centro de Educación Continua del Instituto Politécnico Nacional

Fuente: elaboración propia.

En la figura 6 se muestran las principales razones por las cuales se relacionan los empresarios de los diferentes segmentos del destino turístico de la ciudad de Oaxaca, como se puede observar la principal razón es formar paquetes en conjunto (entre empresas de los diferentes segmentos). Los motivos de relacionarse con empresas de su mismo segmento son: solucionar problemas, pedir apoyo gubernamental, información sobre ocupación y ventas. La relación con el gobierno es con el objetivo de buscar la promoción de su empresa y capacitación. Con las instituciones educativas se relacionan a través de prácticas profesionales, apoyo a la investigación y patrocinio (alojar a alumnos en eventos que se dan en el destino). Y con las asociaciones el principal motivo es buscar ser representados ante el sector turístico y la sociedad de la ciudad de Oaxaca.

\section{Figura 6 \\ PRINCIPALES RAZONES DE COOPERACIÓN LOS EMPRESARIOS TURÍSTICOS}

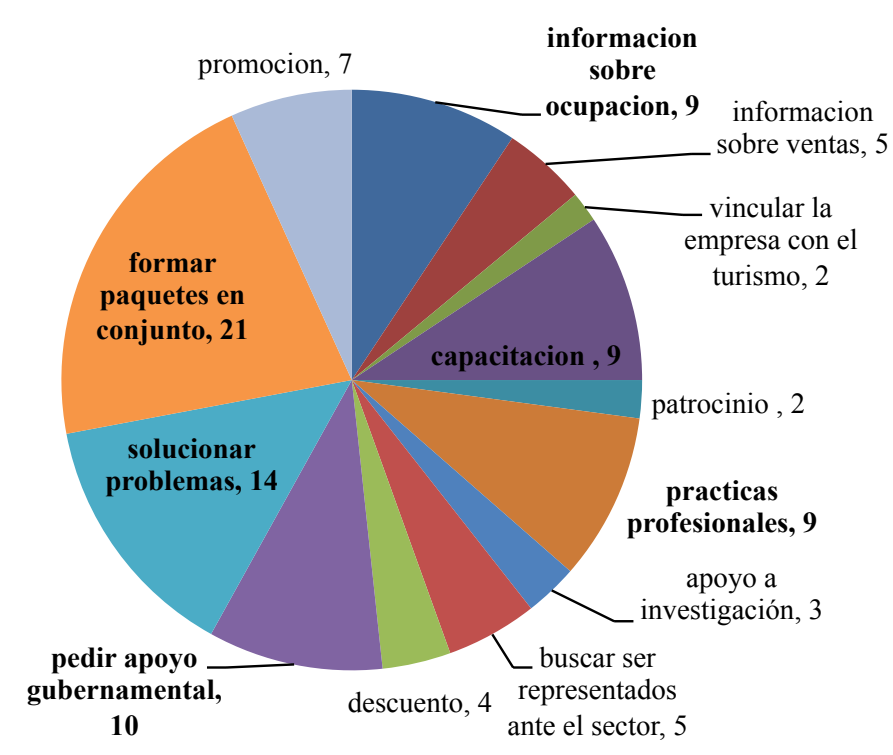

Fuente: elaboración propia. 
En lo que se refiere a la relación que establecen los empresarios turísticos, el 56 $\%$ se relaciona con empresas de su mismo segmento (hotelero-hotelero, restauranterorestaurantero, etc.), con otros segmentos se relacionan en menor proporción (48\%) (hotelero -restaurantero- agencia de viaje, etc.). El $48 \%$ de los empresarios se relaciona con las diferentes áreas del gobierno estatal y municipal. Con las instituciones educativas la vinculación se establece en un $32 \%$.

\section{Figura 7 \\ RED SOCIAL DE LAS ASOCIACIONES TURÍSTICAS DEL DESTINO DE LA CIUDAD DE OAXACA}

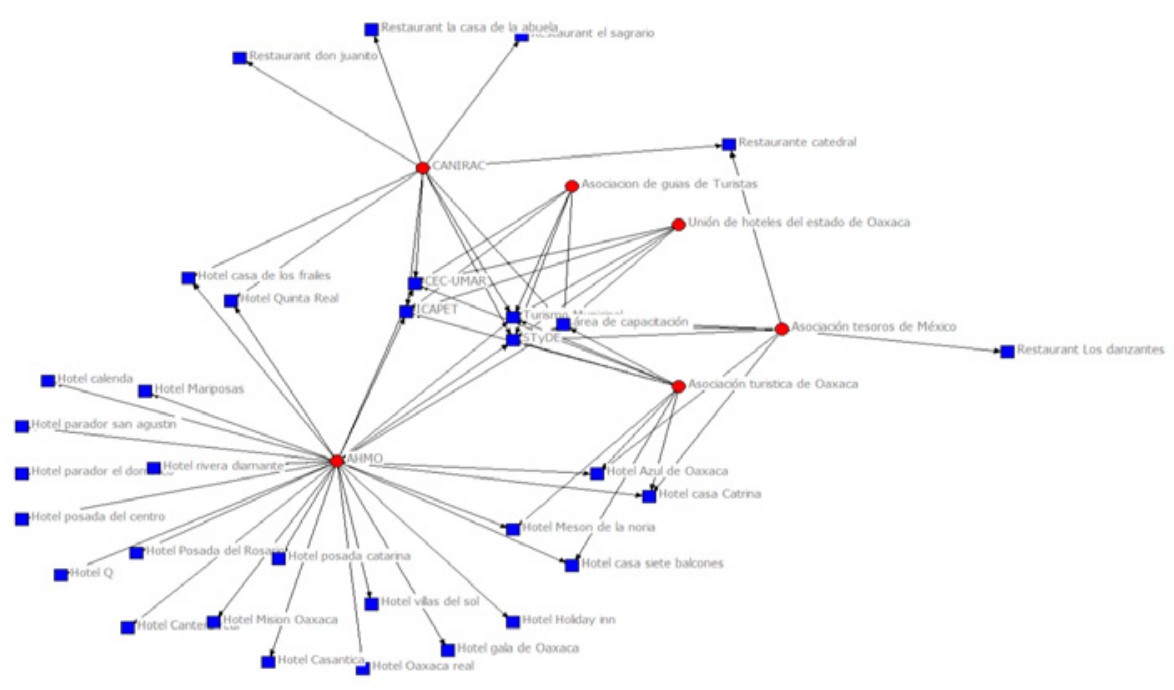

Fuente: elaboración propia.

En lo que respecta a la cooperación del destino, en la figura 7 se observa la red de cooperación entre las asociaciones turísticas. La densidad de esta red es del 10.2\% (muy baja) y está compuesta por subgrupos que son formados por cada una de las agrupaciones. Las asociaciones turísticas en la red de cooperación son fundamentales en la vinculación del gobierno con el empresario turístico y contribuyen a la participación de las empresas en cursos de capacitación y la gestión de la promoción. Así, como para participar en las diferentes convocatorias que emite el gobierno estatal y municipal. También participan como enlace para establecer convenios con instituciones educativas y así vincular a la academia con el sector empresarial turístico. Es de notarse que las organizaciones entrevistadas conciben una visión de falta de compromiso por parte de las instancias gubernamentales, lo que afecta la confianza en las mismas.

En la figura 8 se observa la red de cooperación de las instituciones gubernamentales, y en la misma es de notarse la complejidad de la red que se genera en torno a estas instituciones. Los actores principales son el STyDE, el área de capacitación (de la administración 
turística municipal) y promoción. Las demás áreas como se puede observar juegan un papel menos importante. En esta red de cooperación participan empresarios de todos los segmento turísticos. La densidad de la red formada por las instituciones gubernamentales es del $11.9 \%$ (muy baja).

\section{Figura 8 \\ RED SOCIAL DE LAS INSTITUCIONES GUBERNAMENTALES DEL DESTINO DE LA CIUDAD DE OAXACA}

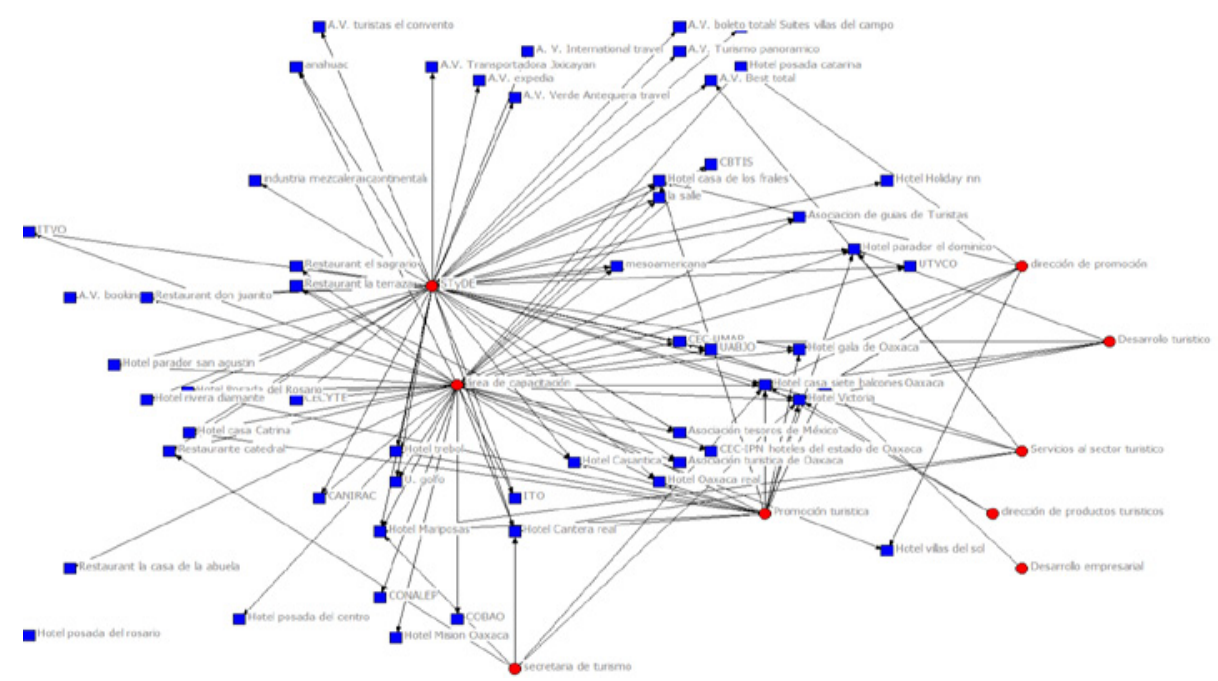

Fuente: elaboración propia.

En la figura 9, se puede observar las instituciones educativas que participan en la red de cooperación del destino de la ciudad de Oaxaca, en donde existen escuelas locales y foráneas. La red que se generó en el análisis es del 3.2\% (muy baja densidad), en donde participan más de 20 instituciones educativas y cada una de ellas coopera con un número reducido de actores. La principal relación se establece por medio de prácticas profesionales (en donde el alumno interactúa con el campo laboral), también a través de brindar información para realizar investigaciones, y son muy pocos los que se vinculan para generar investigaciones de acuerdo a las necesidades de las empresas.

En la figura 10 se representa la red de cooperación de los empresarios turísticos, la cual es considerada como la red global, debido a que los participantes establecen relaciones con todos los demás actores del destino turístico. La densidad de la red de acuerdo al análisis es del 4.4\% (muy baja). En la misma se puede observar el papel central que tiene el gobierno, así como las asociaciones turísticas. El gobierno como impulsor de políticas que contribuyan a la mejora de la prestación de los servicios turísticos, y las asociaciones como enlace entre el gobierno y los empresarios turísticos. Los lazos de cooperación son de carácter económico principalmente (el $60 \%$ de los entrevistados eligieron esta respuesta). La confianza entre los 
empresarios turísticos es baja ya que consideran que pueden ser engañados o ser traicionado al momento de establecer acuerdos de cooperación, también consideran que la información que comparten con otros empresarios puede ser usado en su contra.

Figura 9

\section{RED DE COOPERACIÓN DE LAS INSTITUCIONES EDUCATIVAS DEL DESTINO DE LA CIUDAD DE OAXACA}

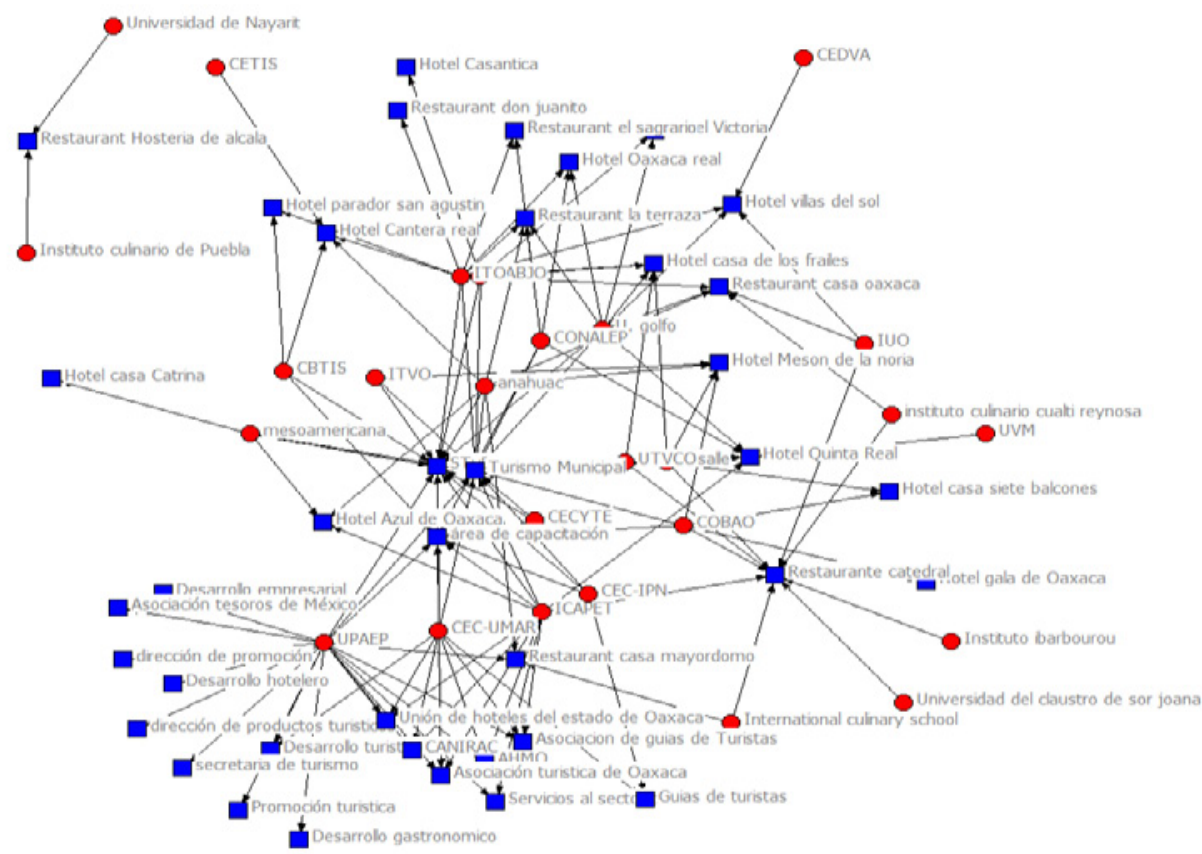

Fuente: elaboración propia.

Por lo que respecta al grado de centralidad, los seis principales actores son: la Secretaria de Turismo y Desarrollo Económico del gobierno del estado (STyDE) (68\%), la Secretaría de Turismo municipal (57\%), la Asociación Mexicana de Hoteles y Moteles (AMHMO) (34\%), Hotel Azul Oaxaca (28\%), restaurante Catedral (22\%), restaurante Hostería de Alcalá (20\%). Este indicador muestra la importancia del papel del gobierno en el destino, así como de la AMHMO y su interacción con los demás actores. Cabe destacar que tres empresas juegan un papel importante en las relaciones que se establecen en la red de cooperación. Para la red en general el grado de centralidad es del $4.13 \%$ (muy bajo), este dato es importante ya que demuestra que el poder de la red no se encuentra en un solo actor.

En el tema de la importancia que tienen los diferentes actores (índice de centralización) el análisis muestra que los diez más importantes son: STyDE, Secretaría de Turismo Municipal, AMHMO, restaurante Catedral, área de capacitación, restaurante hostería de Alcalá, 
hotel parador San Agustín, hotel Casa de los frailes, hotel posada del Centro, hotel Cantera Real. En este indicador se destaca el papel del gobierno, así como de una asociación y de ciertas empresas que tienen mayor peso en la red.

Para el grado de cercanía, figuran la STyDE, el área de capacitación de la Secretaría de Turismo municipal, AMHMO, Hotel Azul de Oaxaca, restaurante Catedral, hotel Casa Catrina, (Instituto Tecnológico de Oaxaca) ITO, hotel los Fraile y CANIRAC. Esto quiere decir que el gobierno, dos asociaciones turísticas, una institución educativa y cuatro prestadores de servicios poseen una mayor capacidad para llegar al resto de los actores.

\section{Figura 10 \\ RED SOCIAL DEL DESTINO TURÍSTICO DE LA CIUDAD DE OAXACA}

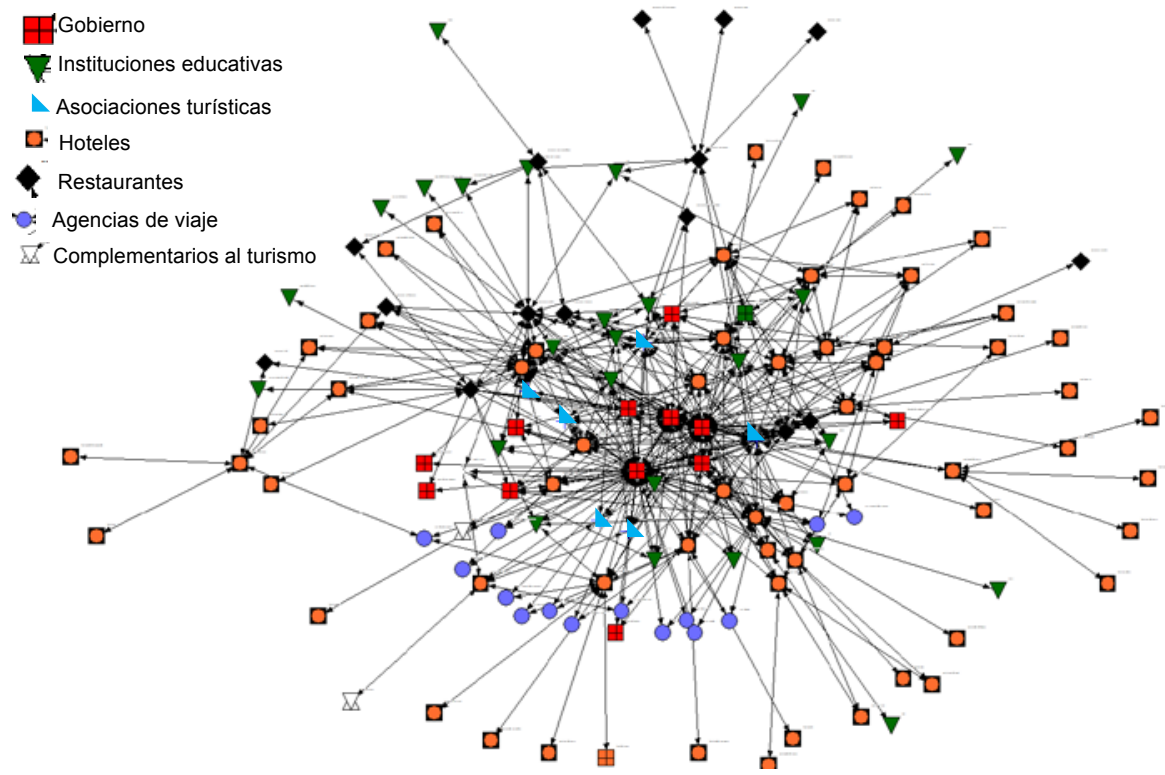

Fuente: elaboración propia.

En resumen, los vínculos incipientes que se relacionan con la densidad de la red social del destino turístico de la ciudad de Oaxaca, indican una muy baja densidad, esto quiere decir que del total de las relaciones que se pueden establecer solo el $4.4 \%$ son efectivas.

\section{CONCLUSIONES}

El destino de la ciudad de Oaxaca, se encuentra en la fase de consolidación del ciclo de vida de los destinos turísticos de Butler, tiene una imagen definida a partir de sus ventajas comparativas que están sustentadas en el gran patrimonio cultural y natural que le permiten competir con otros destinos y estar entre los cuatro primeros lugares en la participación del mercado turístico. 
De acuerdo al resultado obtenido en el análisis de redes sociales turísticas de la ciudad de Oaxaca, el cluster del destino se encuentra en la fase de gestación, esto debido a que los vínculos entre los actores turísticos son incipientes, tanto por la falta de confianza entre ellos mismo (gobierno, instituciones educativas y empresas), como por la baja disposición a cooperar, esto último impulsado por el pensamiento individualista del empresario turístico.

En el destino de la ciudad de Oaxaca aún no se actúa como una red perfectamente vinculada, debido a la falta de interés común en la toma de decisiones, lo que perjudica a los prestadores de servicios. Cada empresario hace lo que cree que es mejor para él, aunque esto no sea lo mejor para el destino. El empresario defiende su forma de trabajar por separado, en detrimento de la calidad del servicio y de su competitividad. Antes que entrar a un programa de mejora, enfocan la mayor parte de sus esfuerzos en poner barreras para que entren otros actores, muchas veces poniendo en peligro la misma viabilidad de la actividad. Lo que genera una serie de obstáculos para el crecimiento y la distribución de la riqueza generada por el turismo.

La información recabada en los foros y en las entrevistas, muestran que en el destino existe desconfianza entre los mismos empresarios, repercutiendo en la poca apertura, flexibilidad y responsabilidad del sector empresarial, limitando la cooperación para trabajar por un beneficio en común. Otro problema interno es la desconfianza hacia agentes de innovación, y escasa inversión en capacitación del personal que labora en las empresas turísticas.

En este proceso, las instituciones gubernamentales tienen un papel central en la red social del cluster, los resultados demuestran la importancia de las mismas en el tejido de las relaciones. La densidad de la red que se conforma es de las más alta entre todos los actores (11.9\%), pero aun así se considera de muy baja densidad. Su poder de relación radica en su papel de formación de habilidades a través de la capacitación y el apoyo en la promoción. Otro actor de suma importancia, son las asociaciones turísticas como intermediarios entre el gobierno y los empresarios turísticos.

El papel de las instituciones educativas está restringido al servicio social y prácticas profesionales, esto resultado de la desconfianza que tienen los demás actores sobre las instituciones educativas.

Las empresas turísticas se vinculan principalmente para formar paquetes en conjunto (con empresas de otros segmentos), y con empresarios de su mismo segmento, para establecer soluciones a sus problemas, y pedir apoyo gubernamental.

La densidad de la red global del destino de la ciudad de Oaxaca es del $4.4 \%$, esto indica que existen vínculos entre los actores turísticos, sin embargo, el número de relaciones aun es incipiente. Esto ligado a la falta de confianza y poca cooperación entre los actores.

Con la información anterior se pueden concluir dos aspectos: primero, el cluster del destino turístico de la ciudad de Oaxaca se encuentra en la fase de gestación, comprobándose la hipótesis propuesta en el presente artículo, ya que las empresas tienen vínculos incipientes aprovechando únicamente las ventajas que le confiere los atractivos culturales del destino. Segundo, el análisis de redes sociales es una herramienta a considerar en el análisis del cluster turístico, ya que permite conocer elementos adicionales a los proporcionados por la información cuantitativa con que se cuenta. 
En lo que se refiere al ciclo de vida del cluster, su desarrollo no es un proceso lineal, ya que se pueden encontrar elementos propuestos en la literatura para cada fase en otras fases. Por ejemplo, si bien el análisis indica que el cluster de la ciudad de Oaxaca está en fase de emergencia, su imagen está bien consolidada en la mente del turista lo que corresponde a elementos de la fase de expansión. En la fase de gestación se tiene como elemento la no existencia de una masa crítica, sin embargo, en este destino si existe una masa crítica, conformada por la presencia de actores especializados en turismo, que corresponde a la fase de expansión.

\section{BIBLIOGRAFÍA}

ACUÑA M., VILLALOBOS D. y RUIZ K. (2000): El cluster ecoturístico de Monteverde/ Costa Rica. Santiago, CEPAL.

BOBBY S. A. (2009): A model for regional technology based economic development, Diseertation doctor of philophy in engineering and applied science.B. S. Louisiana Sate University. USA.

BORGATTI, S.P., EVERETT, M.G. y FREEMAN, L.C. (2002): «Ucinet for Windows: Software for Social Network Analysis», Harvard, MA: Analytic Technologies.

CAI H., y FAN R. (2011): "Analysis of differences in innovation capacity and performance of SMEs cluster», In: Applied economics, Business and Development. International symposium ISAEBD 2011. China.

CASAS, R., DETTMER, J., CELIS, L. y HERNÁNDEZ, C. (2007): «Redes y flujos de conocimiento en la acuacultura mexicana», Redes, vol. 13, no 26, p.111-144.

COITAPO (Comisión de transparencia, acceso a la información pública y protección de datos personales del Estado de Oaxaca) (2014): «Se registran en Oaxaca seis marchas y bloqueos por día», 3 de diciembre de 2014 . Disponible en http://www.buendiatuxtepec.com.mx/en-oaxaca-se-registran-6-marchas-y-bloqueos-a-diario-cotaipo

CRESPO J. (2011): « ¿How emergence conditions of technological clusters affect their viability? Theoretical perspectives on cluster life cycles», European Planning Studies, $\mathrm{n}^{\circ}$. 1, pp. 2025-2046.

CUERVO G. A., MONTORO S.A. y ROMERO M.A. (2009): «Cluster and business innovation», In: Entrepreneurship and business. Galindo M., Guzman J., Ribeiro D. (Eds): A regional perspective Heldelbeg Germany.

DRAGIČEVIĆ M. (2006) : «Regional Clusters as a Source of Competitiveness Building: The case of Croatia», 3rd International Conference - An Enterprise Odyssey: Integration or Disintegration, Zagreb, Croatia. p. 13-148.

DRAGUSIN M., LUMINITA C.D. y PETRESCU R. (2010): «Clustering in transition economies the case romanian tourism industry», Tourism \& hospitality management, pp. 287-301.

EISINGERICH A., BELL S. y TRACERY P. (2010): «How can clusters sustain performance? The role of network strength network openness, and environmental uncertainty», Research policy, nº. 39, pp. 239-253.

EXCELTUR (Alianza para la excelencia turística) (2007). «Plan RENOVE de destinos turísticos españoles», 13 de noviembre de 2014. Madrid. Disponible en http://exceltur.org/ wp-content/uploads/2014/10/PLAN-RENOVE-DESTINOS-TUR\%C3\%8DSTICOSESPA\%C3\%91OLES.pdf 
FERREIRA J. y ESTEVA O. C. (2009): « Regional competitiveness of tourism cluster: a conceptual model proposal», Munich Personal REPEN Archive (MPRA). Consultado el 18 de septiembre de 2014. Disponible en https://mpra.ub.uni-muenchen.de/14853/1/ MPRA_paper_14853.pdf

FILGUEIRAS N. J.M. (2009): «Tres estrategias de marketing para las PyMES del sector turístico oaxaqueño hacia el 2020», Gestión turística, nº. 12, pp.125-140.

GARCÍA G.A. y LARA R.A. (2004): «Cluster y coo-petencia (cooperación y competencia) industrial: algunos elementos teóricos por considerar», Problemas de desarrollo. Revista latinoamericana de economía, vol. 139, nº. 35, pp. 141-162.

GUERRA, L.C. y DANIEL L. (2010): Identificación de oportunidades de negocios para los clusters de mayor impacto en el desarrollo de México. México, Grupo de desarrollo regional del Instituto Tecnológico de Estudios Superiores de Monterrey.

IORDACHE C., CIOCHINA I. y ASANDEI M. (2010): «Clusters tourism activity increase competitiveness support», Theoretical and applied economics, vol.17, no 5, pp. 99-112.

IVANOVIC S., KATIE A. y MIKINAE, K. (2010): «Cluster as a model of sustainable competitiveness of small and medium entrepreneurship in the tourist market», Journal of economics vol.1, no 2, pp. 45-54.

MADRID F.F. y CERÓN M.H. (2013): Evaluación de desempeño de los destinos turísticos en el marco de los convenios de coordinación en materia de reasignación de recursos. México, Universidad Anáhuac-SECTUR.

MANCILDO F.M., REJANE P.N. y SEGRE L. M. (2010): «¿Cuál es el papel del turismo en el desarrollo local? Un análisis crítico del cluster turístico de Santa Teresa- RJ. Brasil», Estudios y perspectivas en turismo, vol. 19, n ${ }^{\circ}$ 5, pp. 812-834.

MÁRQUEZ, S.P.; GONZÁLEZ, P.M.; AGRA, V.Y.; VEGA, N.J.; PINTO, C. A. y QUIROGA, S. E. (2013): «Las redes sociales. Un método para la mejora de la seguridad en las organizaciones sanitarias», Revista española de salud pública, vol. 87, n ० 3 , pp. 209-219.

MENZEL, M.P. y FORNAHL D. (2010): «Cluster life cycles: dimensions and rationales of cluster development», Industrial and Corporate Change, vol.19, pp. 205-238.

MERINO, R. y PULIDO, J.I. (2009): «Desarrollo turístico y dinámica relacional. Metodología de análisis para la gestión activa de destinos turísticos», Cuadernos de Turismo, vol. 23, pp. 173-193.

OAXACA-MÍO. (2000): «Alrededores de la ciudad de Oaxaca», Consultado el 10 de febrero de 2015. Disponible en http://www.oaxaca-mio.com/atrac_turisticos/rutaocotlan.htm

ÖRJAN S. (2008): Clusters equilibrando fuerzas evolutivas y constructivas. Suecia, Danagárds Grafiska.

PORTER M.E. (1998): «Clusters and the new economics of competition», Harvard Business Review, vol. 73, n ${ }^{\circ}$ 6, pp.77-90.

PORTER M.E. (2003): «The economic performance of regions», Regional Studies, vol. $37, \mathrm{n}^{\circ} 6$, pp. 549-578.

REYES, R. y BARRADO, D. (2005): «Nuevas tendencias en el desarrollo de destinos turísticos: marcos conceptuales y operativos para su planificación y gestión», Cuadernos de Turismo, vol. 15, pp. 27-43. 
ROSENFELD S. (1997): «Bringing Business Clusters into the Mainstream of Economic Development», European Planning Studies, vol. 5, n o 1, pp. 3-23.

SANTOS, M. (2010): «Análisis de redes sociales y rendimiento académico, lecciones a partir del caso de los Estados Unidos», Debates en Sociología, vol. 35, pp.7-44.

SANZ, M.L. (2003): «Análisis de redes sociales: o cómo representar las estructuras sociales subyacentes», Apuntes de Ciencia y Tecnología. Vol. 7, pp. 20-30.

SISTEMA DE INFORMACIÓN TURÍSTICA ESTATAL/STYDE OAXACA (2012): «Indicadores básicos de la actividad turística en el estado de Oaxaca». Oaxaca, STyDE.

TOLEDO L. G., QUELOPANA, M. E. M. y CASTROMAN, P. A. (2006): «Estrategia internacional de un cluster turístico latinoamericano», Estudio de caso: Sauipe-Brasil. Consultado el 14 de diciembre de 2014 disponible en: http://www.periodicodeturismo.com.br/site/artigo/pdf/estrategia.PDF

VELÁZQUEZ Á., y AGUILAR G.N. (2005): Manual introductorio al análisis de redes sociales. Medidas de centralidad y ejemplos prácticos con Ucinet 6.85 y Netdraw 1.48, México, Centro de Capacitación y Evaluación para el Desarrollo Rural/Universidad Autónoma de Chapingo

VIVAS J.R. (2001): «Análisis de redes sociales y procesos de influencia en la toma de decisión grupal», Interdisciplinaria, vol. 18, n 1 , pp. 87-113

WILLINER A., SANDOVAL C., FRIAS M. y PÉREZ J. (2012): Redes y pactos sociales territoriales en América Latina y el Caribe: sugerencias metodológicas para su construcción. Santiago, CEPAL 
